\title{
Active contours algorithm with an adaptive gaussian distribution fitting energies and its application to industrial CT image segmentation
}

\author{
Xiao Luo ${ }^{1, a}$, Yongning Zou ${ }^{2}$ \\ ${ }^{1}$ Chongqing University, Chongqing, China \\ ax1233210@163.com,
}

Keywords: Image segmentation; intensity inhomogeneity; Level set method; LBF model; industrial CT images

Abstract. In image segmentation, Local binary fitting model (LBF) is widely used to cope with intensity inhomogeneity. It is insensitive to the initial contour with a large scale parameter and it can make the active contours become smooth and close to the object of the real border with the reduction of scale parameters. For the purpose of enhancing the quality of intensity inhomogeneity image segmentation, we advance a new adaptive rule to obtain the scale parameter. Experimental results on industrial CT image demonstrate that our method is insensitive to the initial contour and accurate for extracting the object.

\section{Introduction}

Image segmentation has always been a focus problem in the field of image analysis and understanding [1-3]. With the help of segmented information, further process such as recognition and reconstruction can be implemented in the area of computer vision. Over the past few decades, researchers have made great efforts in image segmentation and a wide variety of methods have been proposed [4-8].

Active contour model (ACM), as presented in [8-11], has been often used in segmentation with desirable results. An advantage of ACM for image segmentation is that it can provide smooth and closed contours to recover the boundaries of the object. The original active contour model, also called snake model, was introduced in [12] by using explicit curve to extract objects. However, it cannot handle topological changes. To overcome this problem, many methods have been proposed, the PDE-based level set method [16] introduced by Osher and Sethian is the most important and successful one.

Chan-Vese (CV) model [8], as one of the most popular region-based models, is simplified from Mumford-Shah energy functional. By minimizing the energy functional of approximated piecewise constant image, CV model has been successfully applied to images with two regions. However, CV model always assumes that the image intensity is homogeneous in each region. Actually, intensity inhomogeneous images widely exist in the real world $[18,19]$.

In order to solve the limitations of CV model, in [18, 19], by minimizing Mumford-Shah functional [17], Vese and Chan proposed two similar region-based active contour models for the image segmentation. These models, which have been known as piecewise smooth (PS) model, are based on a piecewise smooth description of the images. Therefore, PS model is able to deal with intensity inhomogeneity [15]. However, the computational cost of the PS model is quite expensive, as it involves complicating procedures.

How to take full advantages of local and global regional information is a significant problem. In [22], CV model and LBF model are combined each other with a weighting parameter, which is called LGIF model. By using local and global image information, the robustness to the initialization of the contours can be improved.

The main contribution of this paper is the proposal of a new adaptive rule for gaussian kernel function of LBF model. Under this rule, the value of the scale parameter can be updated dynamically with the energy functional decreases. According to the work in [20], using a large scale parameter, the result of segmentation can be less sensitive to the initialization, and the number of total iterations can be reduced. However, when the scale parameter is small, active contour becomes smooth and approaches the real object boundaries. With the help of this adaptive estimation of the scale parameter, efforts of how to select a proper parameter can be reduced. 
The rest of this paper is organized as follows. In section 2, some well-known region-based models are presented. Then our method is introduced in Section 3. The implementation and results are given in Section 4. Finally this paper is summarized in Section 5.

\section{Existing methods}

Local binary fitting active contour model. To deal with the problem of intensity inhomogeneity in image segmentation, Li et al. proposed the (local binary fitting) LBF model [15, 23]. For a given point $x \in \Omega$, the local intensity fitting energy functional is defined as follows:

$$
\begin{aligned}
E^{L B F}\left(C, f_{1}(x), f_{2}(x)\right)= & \lambda_{1} \int\left[\int_{\text {in }(c)} K_{\sigma}(x-y)\left|I(y)-f_{1}(x)\right|^{2} d y\right] d x+ \\
& \lambda_{2} \int\left[\int_{\text {out }(c)} K_{\sigma}(x-y)\left|I(y)-f_{2}(x)\right|^{2} d y\right] d x
\end{aligned} .
$$

Where $\lambda_{1}$ and $\lambda_{2}$ are positive constants, $f_{1}(x)$ and $f_{2}(x)$ are two smoothing functions which are used to locally approximate image intensities inside and outside contour $C$ respectively. $K_{\sigma}(x-y)$ is the Gaussian kernel function with standard deviation $\sigma$ defined by:

$$
K_{\sigma}(x-y)=\frac{1}{(2 \pi)^{n / 2} \sigma^{n}} \exp \left(-\frac{|x-y|^{2}}{2 \sigma^{2}}\right) \text {. }
$$

In order to penalize the length of the contour [8] and regularize the level set function [23], the two terms can be characterized by the following energy functional:

$$
E^{R}(\phi)=\mu \int_{\Omega} \delta(\phi(x))|\nabla \phi(x)| d x+\int_{\Omega} \frac{1}{2}(|\nabla \phi(x)|-1)^{2} d x .
$$

Therefore, we can define the following energy functional:

$$
E_{\text {all }}^{L B F}\left(\phi, f_{1}(x), f_{2}(x)\right)=E^{L B F}\left(\phi, f_{1}(x), f_{2}(x)\right)+E^{R}(\phi) .
$$

Owing to the introduction of the kernel function, the local region fitting energy of the image is incorporated, thus LBF model is able to provide desirable segmentation results even under the presence of intensity inhomogeneity. However, inappropriate local region information has not always been sufficiently effective and accurate for image segmentation. What's more, LBF model is sensitive to initialization to some extent, which restricts its practical applications.

LGIF model. In [22], the authors combined the advantages of CV model and LBF model by taking the local and global intensity information into account, which is called the LGIF model. In the level set method, this model integrates the local and global intensity fitting energy effectively:

$$
E^{L G I F}\left(\phi, f_{1}, f_{2}, c_{1}, c_{2}\right)=(1-q) E^{L B F}\left(C, f_{1}(x), f_{2}(x)\right)+q E^{C V}\left(C, c_{1}, c_{2}\right) .
$$

Where $q$ is the weighting parameter.

For more accurate computation involving the level set function and penalizing its length, the energy functional is defined by

$$
F\left(\phi, f_{1}, f_{2}, c_{1}, c_{2}\right)=E^{L G I F}\left(\phi, f_{1}, f_{2}, c_{1}, c_{2}\right)+\mu P(\phi)+v L(\phi) .
$$

Where $\mu$ and $v$ are positive constants, and

$$
L(\phi)=\int_{\Omega}|\nabla H(\phi(x))| d x,
$$




$$
P(\phi)=\int_{\Omega} \frac{1}{2}(|\nabla \phi(x)|-1)^{2} d x .
$$

In the LGIF model, the local and global region information is combined by using a weighting parameter $q$. If the intensity inhomogeneity of the image is severe, we select a small $q$. Conversely, we select a relatively large value. Efforts should be made to determine a good estimation of the weighting parameter $q$. What's more, the LGIF model is sensitive to the weighting parameter $q$.

\section{Realization of an adaptive scale coefficient}

As we discussed in section 2, CV model and LBF model have their advantages and disadvantages. LGIF model combines the advantages of CV model and LBF model. Thus, it is natural to use a relatively larger local region to replace the weighted sum of the local region and global region. When the contour is in the neighborhood of object boundaries, we need a small local region to control the moving and stopping of the contour that close to the real object boundaries. On the other hand, when the contour is far away from objects boundaries, we need a relatively large local region to reduce sensitive to initialization.

In order to penalize the length and regularize the level set function, LBF model (4) minimizes the energy functional with respect to $\phi$. And the gradient descent flow:

$$
\frac{\partial \phi}{\partial t}=\delta(\phi)\left(\mu \operatorname{div}\left(\frac{\nabla \phi}{|\nabla \phi|}\right)-\lambda_{1} e_{1}+\lambda_{2} e_{2}\right)+v\left(\nabla^{2} \phi-\operatorname{div}\left(\frac{\nabla \phi}{|\nabla \phi|}\right)\right) .
$$

In LBF model, the kernel function is introduced by Equation (1). To compute the convolutions in [20] more efficiently, the $K_{\sigma}$ can be truncated as a $\omega \times \omega$ mask, where $\omega$ is the smallest odd number no less than $4 \sigma$. Clearly, once the value of kernel function is set, $\omega$ is also determined simultaneously.

Considering the problem of how to select the scale parameter $\sigma$, we propose an adaptive dynamic rule during the process of the contour evolution, which is motivated by the work in [14]. In details, the proposed rule begins with an initial value of $\sigma$, and adaptively updates its value with the level set evolution. According to this method, the energy functional of LBF model will be minimized and we will obtain an automatic estimation of $\sigma$. During the iterative process, we propose the following rule to update the scale parameter $\sigma$ dynamically:

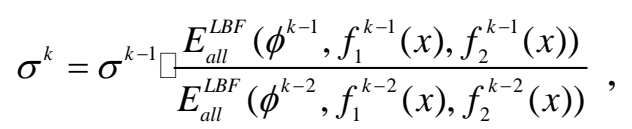

where $k \geq 2, E_{\text {all }}^{L B F}\left(\phi^{k-1}, f_{1}^{k-1}(x), f_{2}^{k-1}(x)\right)$ and $E_{\text {all }}^{L B F}\left(\phi^{k-2}, f_{1}^{k-2}(x), f_{2}^{k-2}(x)\right)$ represent the energy functional of the $(k-1)$ th iteration and the $(k-2)$ th iteration.

To preventing the generation of positive feedback, we judge the trend of the energy functional in Step 4. The algorithm is summarized as follows:

Step 1: Give an original value of the energy functional: $\mathrm{k}=0, \phi^{0}=\phi_{0}(x), \mu, v, \varepsilon, \sigma, w$ and time step $\Delta t$

Step 2: Gets the new value of the following parameters:

$$
f_{1}^{k+1}(x), f_{2}^{k+1}(x), c_{1}^{k+1}(x), c_{2}^{k+1}(x) .
$$

Step 3: Update energy functional $\phi^{k+1}$ by solving (9).

Step 4: If $k \geq 2$ and $\frac{E_{\text {all }}^{L B F}\left(\phi^{k-1}, f_{1}^{k-1}(x), f_{2}^{k-1}(x)\right)}{E_{\text {all }}^{L B F}\left(\phi^{k-2}, f_{1}^{k-2}(x), f_{2}^{k-2}(x)\right)} \leq 1$, update $\sigma^{k+1}$ by (10); else $\sigma^{k+1}=\sigma^{k}, \omega$ is the smallest odd number no less than $4 \sigma$.

Step 5: Check whether the solution is stationary. If not, $\mathrm{k}=\mathrm{k}+1$ and go to step 2. 


\section{Experimental results}

In this section, we compare LBF model [15] and LGIF model [22] with our model on the basis of industrial CT and real-world images. Experimental results are presented to demonstrate the effectiveness of the proposed method. All the experiments were conducted in MATLAB R2012a on a personal computer with Inter Pentium CPU 2.20GHz and 2GB of memory. The level set function $\phi$ can be initialized as a binary step function. Unless otherwise specified, the default parameters are set as $\mu=1.0, \varepsilon=1.0, \Delta t=0.1$, the choices of $\sigma$ and $v$ are different for the test image with various characteristics.

Figure 1 shows a comparison among LBF model, the LGIF model and our method for the real industrial CT image with intensity inhomogeneity. We select $\sigma=3, v=0.002 \times 255 \times 255$ in the first row, $\sigma=3, v=0.002 \times 255 \times 255$ in the second row. We can observe from columns 2 that LBF model fails to segment the object for both of the initial contours, whereas our model works. Table 1 lists the Iterations and the CPU time of Figure 1, which demonstrates that the computational cost of the proposed method is very competitive comparing with other two methods.

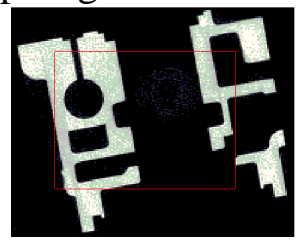

(a)

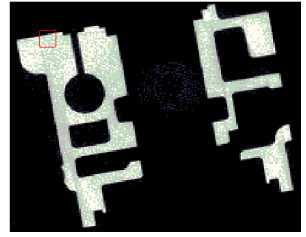

(e)

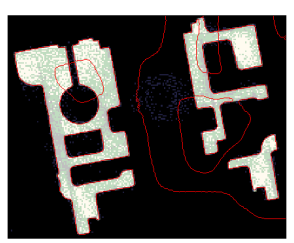

(b)

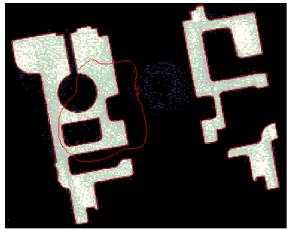

(f)

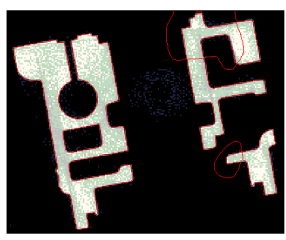

(c)

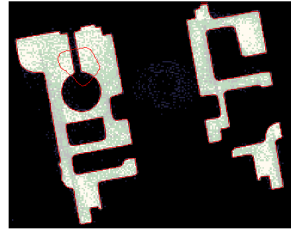

(g)

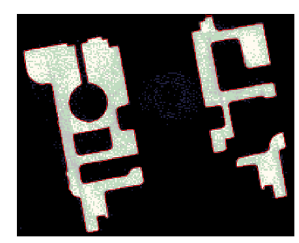

(d)

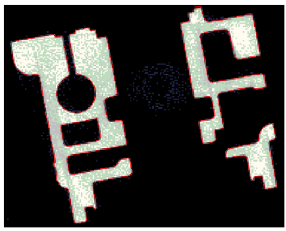

(h)

Figure 1. Comparisons of LBF model, the LGIF model and our method. Column 1: Images with initial contours. Column 2: Results of LBF model [20]. Column 3 Results of the LGIF model [22]. Column 4: Results of our method.

Table 1 Iteration and CPU time of Figure.2

\begin{tabular}{|c|c|c|c|c|c|c|}
\hline & \multicolumn{2}{|c|}{ LBF model } & \multicolumn{2}{|c|}{ LGIF model } & \multicolumn{2}{|c|}{ Our method } \\
\hline & Iterations & time(s) & Iterations & time $(\mathrm{s})$ & Iterations & time $(s)$ \\
\hline Row1 & 50 & 4.695 & 80 & 2.571 & 20 & 1.684 \\
\hline Row2 & 60 & 5.141 & 130 & 6.242 & 30 & 2.456 \\
\hline
\end{tabular}

Real industrial CT images are used to test the effectiveness of our method in practical applications. As comparison, LBF model and the LGIF model are also utilized. And the results are presented in Figure 2. We select the same $\sigma=6, v=0.001 \times 255 \times 255$ in the first and second rows. The last row enlarges the yellow area of the first row. The first column shows the original images. Figures in column 3 show that LGIF model fails to segment the object for both of the initial contours. We can see that similar segmentation consequences are obtained by LBF model and the proposed model. The images in the second row show the consequences that our method is sensitive to the initialization. It is clearly seen from the last row that our method can make active contours closer to the real boundaries of the object. Figure 3 displays the value of the scale parameter and the energy functional change with increasing iterations. We can observe this result consistent with our expectations. 


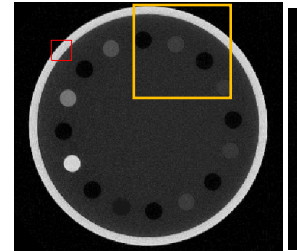

(a)

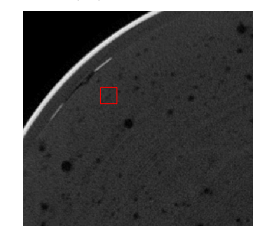

(e)

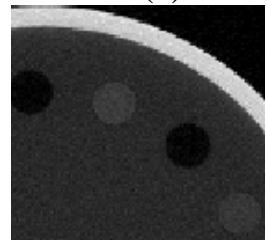

(i)

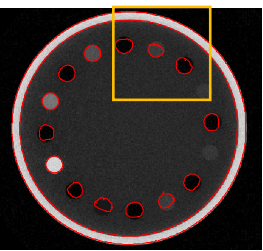

(b)

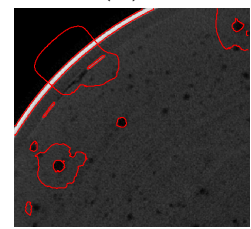

(f)

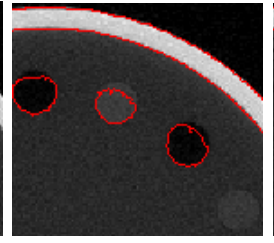

(j)

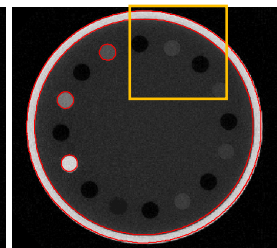

(c)

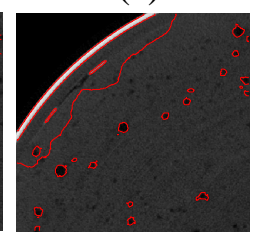

(g)

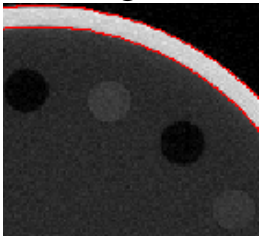

(k)

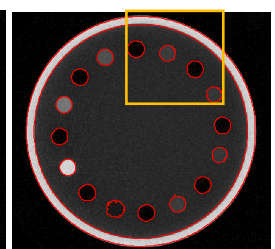

(d)

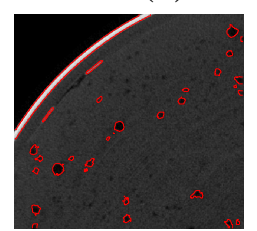

(h)

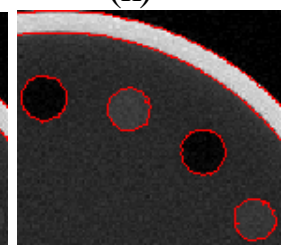

(l)

Figure 2. Results for the industrial CT images with intensity inhomogeneity. Column 1: Images with initial contours. Column 2: Results of LBF model [20]. Column 3: Results of the LGIF model [22]. Column 4: Results of our method.

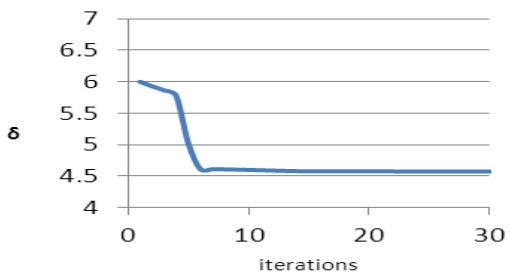

(a)

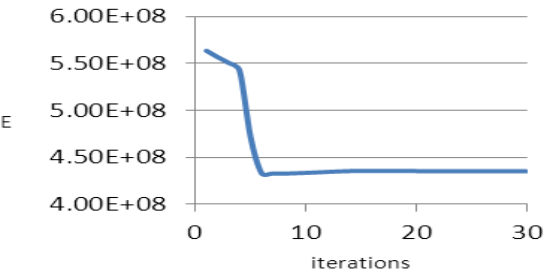

(b)

Figure 3. Value change of the scale parameter and the energy functional with increasing iterations. (a) is the value of the scale parameter change with increasing iterations. (b) is value of the energy functional change with increasing iterations.

\section{Conclusion}

In this paper, our major work is the improvement of the segmentation quality of industrial CT images with intensity inhomogeneity. In our method, the value of the weighting parameter is dynamically updated along with the contour evolution, which is effective and accurate for extracting the object. Comparing with the well-known LBF model, our model not only shows more details, but also much less sensitive to the initial contour. Experimental results demonstrate desirable performance of our extension method for industrial CT images with intensity inhomogeneity.

\section{Acknowledgements}

This research is supported from National major scientific instruments and equipment development special funds (2013YQ030629).

\section{References}

[1] K Zhang, L Zhang, et al. Real-time compressive tracking. European Conference on Computer Vision 2012; 864-877. 
[2] A Asma, W David, et al. On-demand transmission model for remote visualization using image-based rendering. Concurrency and Computation: Practice and Experience 2012; 24(18):2328-2345.

[3] J Ning, L Zhang, et al. Robust mean-shift tracking with corrected background-weighted histogram. IET ComputerVision 2012; 6(1):62-69.

[4] P Hadjidoukas, V Dimakopoulos, et al. A high-performance face detection system using OpenMP. Concurrency and Computation: Practice and Experience 2009; 21(15):1819-1837.

[5] Y Han, W Wang, et al. A new fast multiphase image segmentation algorithm based on nonconvex regularizer. Pattern Recognition 2012; 45(1):363-372.

[6] S Zhu, A Yuille. Region competition: unifying snake/balloon, region growing and Bayes/MDL/energy for multiband image segmentation. IEEE Transactions on Pattern Analysis and Machine Intelligence 1996; 18(9):884-900.

[7] Z Tu, S Zhu. Image segmentation by data-driven Markov chain Monte Carlo. IEEE Transactions on Pattern Analysis and Machine Intelligence 2002; 24(5):657-673.

[8] TF Chan, LA Vese. Active contours without edges. IEEE Trans Image Process 2001;10(2):266-77.

[9] M Kass, A Witkin, Terzopoulos D. Snakes: active contour models. Int J Comput Vision 1988;1(4):321-31.

[10] V Caselles, F Catte, T Coll, F Dibos. A geometric model for active contours in image processing. Numer Math 1993; 66(1):1-31.

[11]Caselles V, Kimmel R, Sapiro G. Geodesic active contours. Int J Comput Vision 1997; 22(1):61-79.

[12]R. Goldenberg, R. Kimmel, E. Rivlin, M. Rudzsky, Fast geodesic active contours, IEEE Transactions on Image Processing 10 (10) (2001) 1467-1475.

[13]J. Park, J.M. Keller, Snakes on the watershed, IEEE Transactions on Pattern Analysis and Machine Intelligence 23 (10) (2001) 1201-1205.

[14] Montefusco LB, Lazzaro D, Papi S. Fast sparse image reconstruction using adaptive nonlinear filtering. IEEE Trans Image Process 2011;20(2):534-44

[15]C. Li, C. Kao, J.C. Gore, Z. Ding, Implicit active contours driven by local binary fitting energy, in: Proceedings of IEEE Conference on Computer Vision and Pattern Recognition, Minneapolis, 2007, pp. 1-7.

[16]S Osher, J.Sethian Fronts propagating with curvature dependent speed: algorithms based on Hamilton-Jacobi formulation. Journal of Computational Physics 1988; 79: 12-49.

[17] D Mumford, J Shah. Optimal approximation by piecewise smooth function and associated variational problems. Communication on Pure and Applied Mathematics 1989; 42:577-685.

[18] L Vese, T Chan. A multiphase level set framework for image segmentation using the Mumford and Shah model. International Journal of Computer Vision 2002; 50(3):271-293.

[19] A Tsai, A Yezzi, et al. Curve evolution implementation of the Mumford-Shah functional for image segmentation, denoising, interpolation, and magnification. IEEE Transaction on Image Processing 2001; 10(8):1169-1186

[20] C Li, C Kao, et al. Minimization of region-scalable fitting energy for image segmentation. IEEE Transaction on Image Processing 2008; 17(10):1940-1949.

[21] S Liu, Y Peng. A local region-based Chan-Vese model for image segmentation. Pattern Recognition 2012; 45(7): 2769-2779. 
[22] L Wang, C Li, Q Sun, D Xia, C Kao. Active contours driven by local and global intensity fitting energy with application to brain MR image segmentation. Comput Med Imaging Graph 2009; 33(7):520-31. 